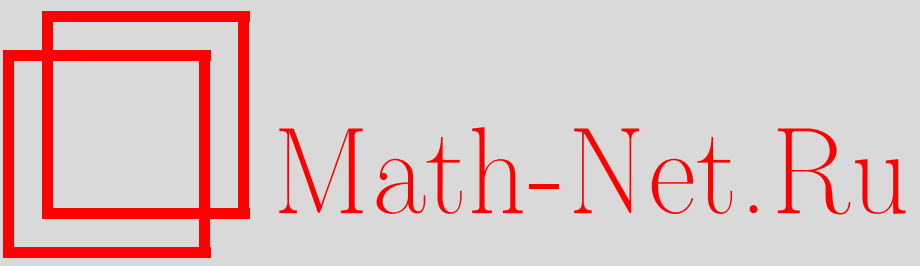

А. В. Полищук, Пропагатор массивного поля спина $s=2$ на пространстве антиде Ситтера, ТМФ, 2004, том 138, номер 1, 81-92

DOI: https://doi.org/10.4213/tmf13

Использование Общероссийского математического портала Math-Net.Ru подразумевает, что вы прочитали и согласны с пользовательским соглашением

http://www.mathnet.ru/rus/agreement

Параметры загрузки:

IP : 18.234 .197 .8

26 апреля 2023 г., 15:07:28 
ТЕОРЕТИЧЕСКАЯ

И МАТЕМАТИЧЕСКАЯ

ФИЗИКА

Том 138, № 1

январь, 2004

(C) 2004 r.

\author{
А.В. Полишук ${ }^{*}$
}

\title{
ПРОПАГАТОР МАССИВНОГО ПОЛЯ СПИНА $s=2$ НА ПРОСТРАНСТВЕ АНТИ-ДЕ СИТТЕРА
}

Построен пропагатор массивного тензорного поля второго ранга на евклидовом продолжении пространства анти-де Ситтера (AdS). Найдено явное выражение для пропагатора в пределе, когда поле принимает значение на границе пространства AdS. Показано, что предельное выражение правильно воспроизводит функцию Грина и двухточечную корреляционную функцию граничной конформной теории поля, как и предсказывает гипотеза AdS/CFT-соответствия. Получено еще одно свидетельство в пользу интерпретации операторов граничной конформной теории поля как определенных пределов квантовых полей, распространяющихся в пространстве AdS.

Ключевые слова: функция Грина, конформная теория поля, AdS/CFT-соответствие.

\section{1. ВВЕДЕНИЕ}

В связи с предложенной недавно гипотезой AdS/CFT-соответствия, сопоставляюшей теории супергравитации на пространстве анти-де Ситтера (AdS) некоторую конформную теорию поля (КTП) на его гранище, развернулась активная деятельность по изучению теоретико-полевых аспектов пространства AdS (см. обзор [1]). В частности, значительные усилия были направлены на вычисление всевозможных корреляционных функций операторов, соответствующих полям, которые образуют полный спектр IIB супергравитации, возмущенной относительно фонового пространства $A d S_{5} \times S^{5}$. Вместе с тем многие результаты, касаюшиеся квантовой теории поля на пространствах анти-де Ситтера и де Ситтера, были получены много лет назад. Так, например, пропагаторы для скалярных [2]-[4], спинорных [5] и векторных [6] полей были построены при произвольных значениях масс, а пропагаторы для калибровочных бозонов и гравитонньй пропагатор были найдены в ковариантных калибровках в работах [6]-[9]. С появлением гипотезы AdS/CFT-соответствия вновь были выведены пропагаторы для калибровочных бозонов и гравитонов, при этом в работе [10] была использована нековариантная калибровка, а ковариантное рассмотрение было представлено в работах [11], [12].

В настоящей статье мы продолжим исследование теории поля на пространстве AdS, вычислив пропагатор массивного тензорного поля второго ранга. Данное поле присутствует в свободном спектре IIB супергравитации на пространстве $A d S_{5} \times S^{5}$ с массой

\footnotetext{
* Математический институт им. В. А. Стеклова PAН, Москва, Россия. E-mail: alexey@mi.ras.ru
} 
$m^{2}=k(k+4) e^{2}$, где $k=1,2, \ldots$, а $e$ - радиус AdS [13]. Наше вычисление основывается на методе битензорного разложения, впервые предложенном в работе [6], где были найдены пропагаторы для векторных и калибровочных полей. Данный метод основывается на том обстоятельстве, что любой пропагатор на пространстве AdS должен удовлетворять всем изометриям пространства и, таким образом, всегда может быть выражен через единственную переменную - хордовое расстояние в пространстве вложения. С геометрической точки зрения пропагатор есть битензор, т.е. тензор, который зависит от координат двух различных точек и несет тензорные индексы, отвечаюшие касательным пространствам в этих точках. Таким образом, пропагатор может быть представлен в виде разложения по базису максимально симметрических фундаментальных (МСФ) битензоров, возникаюших в результате ковариантного дифференцирования хордового расстояния. Структура МСФ-битензоров, используемых в разложении, определяется симметриями соответствуюшего пропагатора. Заметим, что существование МС $\Phi$-битензоров является отличительной особенностью максимально симметрических пространств, т.е. пространств, обладающих максимальным числом векторов Киллинга. Подстановка пропагатора в виде разложения по МСФ-битензорам в уравнение движения дает систему обычных дифференциальных уравнений для скалярных функций. Решение такой системы определяется однозначно вследствие наложения граничных условий на больших и малых расстояниях. С помощью этой процедуры нам удается построить пропагатор для массивного тензорного поля спина $s=2$. Оказывается, что он может быть представлен в терминах единственной скалярной функции от хордового расстояния. Кроме того, при стремлении массы поля к нулю мы получаем калибровочно-инвариантный вклад в пропагатор гравитона, найденного в работе [12]. В пределе, когда массивное поле принимает значение на гранище пространства AdS, полученный пропагатор правильно воспроизводит как функцию Грина, вычисленную ранее в работах [14], [15], так и двухточечную корреляционную функцию в граничной КТП. В результате нам удается установить соотношение между массивным полем спина $s=2$ в супергравитации на фоновом пространстве AdS и составным примарным киральным оператором в граничной КТП. Отметим, что полученное выражение для пропагатора справедливо при произвольных значениях размерности пространства $\mathrm{AdS}$ и массы поля.

Работа построена следующим образом. В разделе 2 описывается стандартная параметризация евклидова продолжения пространства AdS и строится пропагатор для массивного симметрического тензорного поля. Раздел 3 посвящен анализу поведения полученного пропагатора в пределе, когда массивное поле принимает значение на границе пространства. Также здесь устанавливается соотношение между массивным симметрическим тензорным полем, распространяюшимся на пространстве AdS, и оператором в граничной КТП. В разделе 4 обсуждаются полученные результаты.

\section{2. ПРОПАГАТОР МАССИВНОГО ПОЛЯ СПИНА $s=2$}

Евклидово продолжение пространства $A d S_{d+1}$ можно представить как подпространство, где $Y_{-1}>0$, гиперболоида:

$$
Y^{2} \equiv-\left(Y_{-1}\right)^{2}+\left(Y_{0}\right)^{2}+\sum_{i=1}^{d}\left(Y_{i}\right)^{2}=-\frac{1}{e^{2}},
$$


где $e$ - радиус $A d S_{d+1}$. Если ввести параметризацию Пуанкаре

$$
x_{i}=\frac{Y_{i}}{e\left(Y_{0}+Y_{-1}\right)}, \quad x_{0}=\frac{1}{e^{2}\left(Y_{0}+Y_{-1}\right)},
$$

то индуцированная на гиперболоиде метрика совпадет с метрикой пространства Лобачевского

$$
d s^{2}=-\left(d Y_{-1}\right)^{2}+\left(d Y_{0}\right)^{2}+\sum_{i=1}^{d}\left(d Y_{i}\right)^{2}=\frac{1}{e^{2} x_{0}^{2}}\left(d x^{0} d x^{0}+\sum_{i=1}^{d} d x^{i} d x^{i}\right),
$$

причем $x_{0} \geqslant 0$. Граница пространства включает гиперплоскость $x_{0}=0$ и бесконечно удаленную точку $x_{0}=\infty$. В дальнейшем мы положим радиус пространства AdS paвным единице, так что скалярная кривизна пространства станет равной $\mathcal{R}=-d(d+1)$. Следуя методу, изложенному в работе [11], введем переменную $u$, отвечающую расстоянию между двумя произвольными точками $x(Y)$ и $y\left(Y^{\prime}\right)$ в пространстве вложения, следуюшим образом:

$$
u \equiv \frac{1}{2}\left(Y-Y^{\prime}\right)^{2}=\frac{(x-y)^{2}}{2 x_{0} y_{0}},
$$

где $(x-y)^{2}=\delta_{\mu \nu}(x-y)_{\mu}(x-y)_{\nu}$. Частные производные от $u$ равны

$$
\begin{aligned}
\partial_{\mu} u & =\frac{1}{x_{0}}\left[\frac{1}{y_{0}}(x-y)_{\mu}-u \delta_{\mu 0}\right], \\
\partial_{\nu^{\prime}} u & =\frac{1}{y_{0}}\left[\frac{1}{x_{0}}(y-x)_{\nu^{\prime}}-u \delta_{\nu^{\prime} 0}\right] .
\end{aligned}
$$

Как было отмечено во введении, все инвариантные функции на пространстве AdS могут быть представлены в терминах этой переменной и ее ковариантных производных. В дальнейшем мы воспользуемся некоторыми свойствами различных производных от $u$, которые мы приводим ниже [11]:

$$
\begin{gathered}
\square u=D^{\mu} \partial_{\mu} u=(d+1)(1+u), \\
D^{\mu} u \partial_{\mu} u=u(2+u), \\
D_{\mu} \partial_{\nu} u=g_{\mu \nu}(1+u), \\
\left(D^{\mu} u\right)\left(D_{\mu} \partial_{\nu} \partial_{\nu^{\prime}} u\right)=\partial_{\nu} u \partial_{\nu^{\prime}} u, \\
\left(D^{\mu} u\right)\left(\partial_{\mu} \partial_{\nu^{\prime}} u\right)=(1+u) \partial_{\nu^{\prime}} u, \\
\left(D^{\mu} \partial_{\mu^{\prime}} u\right)\left(\partial_{\mu} \partial_{\nu^{\prime}} u\right)=g_{\mu^{\prime} \nu^{\prime}}+\partial_{\mu^{\prime}} u \partial_{\nu^{\prime}} u, \\
D_{\mu} \partial_{\nu} \partial_{\nu^{\prime}} u=g_{\mu \nu} \partial_{\nu^{\prime}} u .
\end{gathered}
$$

Свободные уравнения движения для массивного поля спина $s=2$ на пространстве, являюшемся пространством Эйнштейна, имеют следуюший вид [13]:

$$
\begin{gathered}
D^{\sigma} D_{\sigma} \phi_{\mu \nu}+\left(2-m^{2}\right) \phi_{\mu \nu}=0, \\
D^{\sigma} \phi_{\sigma \mu}=0, \quad \phi_{\sigma}^{\sigma}=0 .
\end{gathered}
$$


Отметим, что уравнения (4), (5) могут быть получены из единого уравнения второго порядка [16]

$$
\begin{array}{r}
D^{\sigma} D_{\sigma} \phi_{\mu \nu}+\left(2-m^{2}\right) \phi_{\mu \nu}-D_{\mu} D^{\sigma} \phi_{\sigma \nu}-D_{\nu} D^{\sigma} \phi_{\sigma \mu}+D_{\mu} D_{\nu} \phi_{\sigma}^{\sigma}- \\
-2 g_{\mu \nu} \phi_{\sigma}^{\sigma}+g_{\mu \nu}\left(D^{\sigma} D^{\rho} \phi_{\sigma \rho}-D^{\rho} D_{\rho} \phi_{\sigma}^{\sigma}+d \phi_{\sigma}^{\sigma}+m^{2} \phi_{\sigma}^{\sigma}\right)=0 .
\end{array}
$$

Данное уравнение содержит связи, отвечаюшие бесследовости и поперечности тензора $\phi_{\mu \nu}$, а однозначность его выбора обусловлена тем, что при $d=4$ и $m^{2}=k(k+4)$ оно воспроизводит свободную часть уравнений теории IIB супергравитации, компактифицированной на $S^{5}$, для массивных мод метрики, возмушенной относительно метрики $A d S_{5}$. Следуя работе [16], покажем, как из уравнения (6) получается система (4), (5). Обозначив левую часть уравнения (6) символом $E_{\mu \nu}$, получаем следуюшие тождества [16]:

$$
\begin{aligned}
& 0=D^{\mu} E_{\mu \nu}=-m^{2} D^{\mu} \phi_{\mu \nu}+m^{2} D_{\nu} \phi_{\sigma}^{\sigma}, \\
& 0=\frac{m^{2}}{d-1} g^{\mu \nu} E_{\mu \nu}+D^{\mu} D^{\nu} E_{\mu \nu}=\frac{d m^{2}}{d-1}\left(m^{2}-d-1\right) \phi_{\sigma}^{\sigma} .
\end{aligned}
$$

Таким образом, видно, что уравнения (5) действительно содержатся в уравнении (6). Окончательно, подставляя (5) в (6), получаем уравнение (4).

Для вычисления пропагатора поля $\phi_{\mu \nu}$ мы добавляем в уравнения движения внешний источник $T_{\mu \nu}$. Для того чтобы выполнялись уравнения связи (5), нужно потребовать, чтобы тензор $T_{\mu \nu}$ был поперечным и бесследовым. Как обычно, представим решение уравнения (6) с внешним источником в правой части в виде

$$
\phi_{\mu \nu}(x)=\int d^{d+1} y \sqrt{g} G_{\mu \nu ; \mu^{\prime} \nu^{\prime}}(x, y) T^{\mu^{\prime} \nu^{\prime}}(y),
$$

где $G_{\mu \nu ; \mu^{\prime} \nu^{\prime}}(x, y)$ является пропагатором поля $\phi_{\mu \nu}$ и, следовательно, удовлетворяет неоднородному дифференциальному уравнению

$$
\begin{aligned}
D^{\sigma} D_{\sigma} & G_{\mu \nu ; \mu^{\prime} \nu^{\prime}}+\left(2-m^{2}\right) G_{\mu \nu ; \mu^{\prime} \nu^{\prime}}-D_{\mu} D^{\sigma} G_{\sigma \nu ; \mu^{\prime} \nu^{\prime}}- \\
& \quad-D_{\nu} D^{\sigma} G_{\sigma \mu ; \mu^{\prime} \nu^{\prime}}+D_{\mu} D_{\nu} G_{\sigma ; \mu^{\prime} \nu^{\prime}}^{\sigma}-2 g_{\mu \nu} G_{\sigma ; \mu^{\prime} \nu^{\prime}}^{\sigma}+ \\
& \quad+g_{\mu \nu}\left(D^{\sigma} D^{\rho} G_{\sigma \rho ; \mu^{\prime} \nu^{\prime}}-D^{\rho} D_{\rho} G_{\sigma ; \mu^{\prime} \nu^{\prime}}^{\sigma}+d G_{\sigma ; \mu^{\prime} \nu^{\prime}}^{\sigma}+m^{2} G_{\sigma ; \mu^{\prime} \nu^{\prime}}^{\sigma}\right)= \\
= & \frac{1}{\sqrt{g}}\left(g_{\mu \mu^{\prime}} g_{\nu \nu^{\prime}}+g_{\mu \nu^{\prime}} g_{\nu \mu^{\prime}}\right) \delta(x, y) .
\end{aligned}
$$

Заметим, что $G_{\mu \nu ; \mu^{\prime} \nu^{\prime}}(x, y)$ является бесследовым поперечным тензором с точностью до локальных членов

$$
\begin{aligned}
G_{\sigma ; \mu^{\prime} \nu^{\prime}}^{\sigma}= & \frac{d-1}{d m^{2}\left(m^{2}-d-1\right)} \frac{1}{\sqrt{g}}\left(\frac{2 m^{2}}{d-1} g_{\mu^{\prime} \nu^{\prime}}+D_{\mu^{\prime}} D_{\nu^{\prime}}+D_{\nu^{\prime}} D_{\mu^{\prime}}\right) \delta(x, y) \\
D^{\sigma} G_{\sigma \nu ; \mu^{\prime} \nu^{\prime}}= & \frac{d-1}{d m^{2}\left(m^{2}-d-1\right)} \frac{1}{\sqrt{g}}\left(\frac{2 m^{2}}{d-1} g_{\mu^{\prime} \nu^{\prime}}+D_{\mu^{\prime}} D_{\nu^{\prime}}+D_{\nu^{\prime}} D_{\mu^{\prime}}\right) D_{\nu} \delta(x, y)- \\
& -\frac{1}{m^{2}} \frac{1}{\sqrt{g}}\left(g_{\nu \mu^{\prime}} D_{\nu^{\prime}}+g_{\nu \nu^{\prime}} D_{\mu^{\prime}}\right) \delta(x, y)
\end{aligned}
$$


Однако следует отметить, что структура локальных членов и свойства тензора $T_{\mu^{\prime} \nu^{\prime}}$ приводят к тому, что выражение (7) действительно удовлетворяет уравнениям связи (5).

Представим пропагатор $G_{\mu \nu: \mu^{\prime} \nu^{\prime}}(x, y)$ в виде разложения по МС $\Phi$-битензорам:

$$
\begin{aligned}
G_{\mu \nu ; \mu^{\prime} \nu^{\prime}}= & T_{\mu \nu ; \mu^{\prime} \nu^{\prime}}^{(1)} A(u)+T_{\mu \nu ; \mu^{\prime} \nu^{\prime}}^{(2)} B(u)+T_{\mu \nu ; \mu^{\prime} \nu^{\prime}}^{(3)} C(u)+ \\
& +T_{\mu \nu ; \mu^{\prime} \nu^{\prime}}^{(4)} D(u)+T_{\mu \nu ; \mu^{\prime} \nu^{\prime}}^{(5)} E(u),
\end{aligned}
$$

где

$$
\begin{aligned}
& T_{\mu \nu ; \mu^{\prime} \nu^{\prime}}^{(1)}=g_{\mu \nu} g_{\mu^{\prime} \nu^{\prime}}, \\
& T_{\mu \nu ; \mu^{\prime} \nu^{\prime}}^{(2)}=\partial_{\mu} u \partial_{\nu} u \partial_{\mu^{\prime}} u \partial_{\nu^{\prime}} u, \\
& T_{\mu \nu ; \mu^{\prime} \nu^{\prime}}^{(3)}=\partial_{\mu} \partial_{\mu^{\prime}} u \partial_{\nu} \partial_{\nu^{\prime}} u+\partial_{\mu} \partial_{\nu^{\prime}} u \partial_{\nu} \partial_{\mu^{\prime}} u, \\
& T_{\mu \nu ; \mu^{\prime} \nu^{\prime}}^{(4)}=g_{\mu \nu} \partial_{\mu^{\prime}} u \partial_{\nu^{\prime}} u+g_{\mu^{\prime} \nu^{\prime}} \partial_{\mu} u \partial_{\nu} u, \\
& T_{\mu \nu ; \mu^{\prime} \nu^{\prime}}^{(5)}=\partial_{\mu} \partial_{\mu^{\prime}} u \partial_{\nu} u \partial_{\nu^{\prime}} u+\partial_{\nu} \partial_{\mu^{\prime}} u \partial_{\mu} u \partial_{\nu^{\prime}} u+\left(\mu^{\prime} \leftrightarrow \nu^{\prime}\right) .
\end{aligned}
$$

Все выписанные битензоры симметричны относительно замены $\mu \leftrightarrow \nu, \mu^{\prime} \leftrightarrow \nu^{\prime}$, а также относительно перестановки $(\mu \nu) \leftrightarrow\left(\mu^{\prime} \nu^{\prime}\right)$, что отвечает симметриям тензора $G_{\mu \nu ; \mu^{\prime} \nu^{\prime}}$. Для удобства дальнейших вычислений перегруппируем члены в выражении (11) таким образом, чтобы выделить полные производные:

$$
\begin{aligned}
G_{\mu \nu ; \mu^{\prime} \nu^{\prime}}= & \left.\partial_{\mu} \partial_{\mu^{\prime}} u \partial_{\nu} \partial_{\nu^{\prime}} u+\partial_{\mu} \partial_{\nu^{\prime}} u \partial_{\nu} \partial_{\mu^{\prime}} u\right) G(u)+g_{\mu \nu} g_{\mu^{\prime} \nu^{\prime}} H(u)+ \\
& +D_{(\mu}\left[\partial_{\nu)} \partial_{\mu^{\prime}} u \partial_{\nu^{\prime}} u X(u)\right]+D_{\left(\mu^{\prime}\right.}\left[\partial_{\left.\nu^{\prime}\right)} \partial_{\mu} u \partial_{\nu} u X(u)\right]+ \\
& +D_{(\mu}\left[\partial_{\nu)} u \partial_{\mu^{\prime}} u \partial_{\nu^{\prime}} u Y(u)\right]+D_{\left(\mu^{\prime}\right.}\left[\partial_{\left.\nu^{\prime}\right)} u \partial_{\mu} u \partial_{\nu} u Y(u)\right]+ \\
& +D_{\mu}\left[\partial_{\nu} u Z(u)\right] g_{\mu^{\prime} \nu^{\prime}}+D_{\mu^{\prime}}\left[\partial_{\nu^{\prime}} u Z(u)\right] g_{\mu \nu} .
\end{aligned}
$$

Представления (11) и (13) для $G_{\mu \nu ; \mu^{\prime} \nu^{\prime}}$ являются эквивалентными, поскольку фигурирующие в них скалярные функции связаны друг с другом простым преобразованием:

$$
\begin{gathered}
A=H+2(1+u) Z, \quad B=2 Y^{\prime}, \quad C=G+2 X, \\
D=2 X+(1+u) Y+Z^{\prime}, \quad E=X^{\prime}+Y,
\end{gathered}
$$

которое, очевидно, обратимо. Таким образом, решение уравнения (8) может быть выписано в любом из двух базисов (11), (13).

Подстановка тензора $G_{\mu \nu ; \mu^{\prime} \nu^{\prime}}$ в форме (13) в уравнение (8) и приравнивание нулю выражений, возникаюших перед каждым независимым МС $\Phi$-битензором, приводит к следующей системе дифференциальных уравнений на коэффициентные функции $G, H$, $X, Y$ и $Z$ :

$$
\begin{aligned}
& 2 G^{\prime \prime}-(1+u)(d-1) Y^{\prime \prime}-2\left(d-1+m^{2}\right) Y^{\prime}-2(d-1) X^{\prime \prime}+(d-1) Z^{\prime \prime \prime}=0 \\
& 2 G^{\prime \prime}+(d-1) H^{\prime \prime}+(d-1)(1+u) Z^{\prime \prime}-2(d-1)(1+u) X^{\prime}-(d-1)(1+u)^{2} Y^{\prime}+ \\
& \quad+\left(2 d-2-m^{2}\right) Z^{\prime}-2\left(2 d-2+m^{2}\right) X-\left(3 d-3+m^{2}\right)(1+u) Y=0 \\
& (1+u) \\
& \quad G^{\prime \prime}+(d-1) G^{\prime}+\left(2 d-2+m^{2}\right) X^{\prime}+(d-1)(1+u) Y^{\prime}+ \\
& \quad+\left(d-1+m^{2}\right) Y-(d-1) Z^{\prime \prime}=0 \\
& u(2+u) G^{\prime \prime}+(d-1)(1+u) G^{\prime}+\left(2-2 d-m^{2}\right) G+(d-1) Z^{\prime}-(d-1)(1+u) Y+ \\
& \quad+2\left(1-d-m^{2}\right) X=\frac{1}{\sqrt{g}} \delta(x, y) .
\end{aligned}
$$


Сделаем некоторые замечания относительно выписанной системы. Уравнения (15)-(18) соответствуют битензорам $T_{\mu \nu ; \mu^{\prime} \nu^{\prime}}^{(2)}, T_{\mu \nu ; \mu^{\prime} \nu^{\prime}}^{(3)}, T_{\mu \nu ; \mu^{\prime} \nu^{\prime}}^{(4)}$ и $T_{\mu \nu ; \mu^{\prime} \nu^{\prime}}^{(5)}$, в то время как уравнения, соответствуюшие битензорам $T_{\mu \nu ; \mu^{\prime} \nu^{\prime}}^{(1)}$ и $g_{\mu \nu} \partial_{\mu^{\prime}} u \partial_{\nu^{\prime}} u$, являются зависимыми и, следовательно, не будут участвовать в последующих рассмотрениях. Интегрируя уравнение (15), получаем

$$
2 G^{\prime}-(1+u)(d-1) Y^{\prime}-\left(d-1+2 m^{2}\right) Y-2(d-1) X^{\prime}+(d-1) Z^{\prime \prime}=0 .
$$

Складывая (17) и (19), находим

$$
(1+u) G^{\prime \prime}+(d+1) G^{\prime}+m^{2} X^{\prime}-m^{2} Y=0 .
$$

Далее, интегрирование (17) с использованием (20) дает

$$
2(1+u) G^{\prime}+2(d-1) G+2\left(d-1+m^{2}\right) X+(d-1)(1+u) Y-(d-1) Z^{\prime}=0 .
$$

Складывая теперь (18) и (21), получаем уравнение второго порядка на функцию $G$ :

$$
u(2+u) G^{\prime \prime}+(d+1)(1+u) G^{\prime}-m^{2} G=\frac{1}{\sqrt{g}} \delta(x, y) .
$$

Решение этого уравнения однозначно определяется тем условием, что сингулярность при $u \rightarrow 0$ должна совпадать с сингулярностью в случае плоского пространства, а также требованием быстрого убывания на бесконечности $(u \rightarrow \infty)$. В результате решение для функции $G(u)$ дается формулой

$$
G(u)=\frac{\Gamma(\Delta)}{2^{\Delta+1} \pi^{d / 2} \Gamma(\Delta-d / 2+1)} z^{-\Delta}{ }_{2} F_{1}\left(\frac{\Delta}{2}, \frac{\Delta+1}{2} ; \Delta-\frac{d}{2}+1 ; z^{-2}\right),
$$

где $z=1+u$ и

$$
\Delta=\frac{d}{2}+\sqrt{\left(\frac{d}{2}\right)^{2}+m^{2}} .
$$

Сконцентрируем наше внимание на функциях $X, Y, Z$ и $H$. С этой целью воспользуемся соотношением (9) и разложим $G_{\mu \nu ; \mu^{\prime} \nu^{\prime}}$ по базису фундаментальных битензоров, приравнивая выражение, стояшее перед структурой $\partial_{\mu^{\prime}} u \partial_{\nu^{\prime}} u$, к нулю:

$$
2 G+4(1+u) X^{\prime}+2(d+3) X+2 u(2+u) Y^{\prime}+(d+5)(1+u) Y+(d+1) Z^{\prime}=\mathcal{F} .
$$

Здесь $\mathcal{F}(u)$ обозначает функцию, возникаюшую перед $\partial_{\mu^{\prime}} u \partial_{\nu^{\prime}} u$ в разложении правой части (9) по битензорам. Аналогично, раскладывая $G_{\mu \nu ; \mu^{\prime} \nu^{\prime}}$ и подставляя в соотношение (10), находим

$$
2 u(2+u) Y^{\prime \prime}+(2 d+11)(1+u) Y^{\prime}+(2 d+7) Y+2(1+u) X^{\prime \prime}+2(d+4) X^{\prime}+Z^{\prime \prime}=\mathcal{F}^{\prime} .
$$


В данном случае мы ограничились рассмотрением фундаментального битензора $\partial_{\nu} u \partial_{\mu^{\prime}} u \partial_{\nu^{\prime}} u$. Далее, разрешая уравнения (18) и (24) относительно $Z^{\prime}$ и принимая во внимание (22), получаем

$$
\begin{aligned}
(d+1) & (1+u) G^{\prime}+(d+2)(d-1) G+2(d-1)(1+u) X^{\prime}+ \\
& +\left(2(d+2)(d-1)+m^{2}(d+1)\right) X+ \\
& +(d-1) u(2+u) Y^{\prime}+(d+3)(d-1)(1+u) Y=\frac{d-1}{2} \mathcal{F} .
\end{aligned}
$$

Дифференцирование уравнения (21), а затем использование (25) с последующим интегрированием по переменной $u$ дает

$$
\begin{gathered}
(d-1)\left(u(2+u) Y^{\prime}+(d+4)(1+u) Y+(1+u) X^{\prime}+(d+4) X\right)+ \\
+m^{2} X+(1+u) G^{\prime}+(d-1) G=\frac{d-1}{2} \mathcal{F}
\end{gathered}
$$

Вычитая (25) из (27) и принимая во внимание (20), находим следуюшее выражение для функции $X$ :

$$
X=\frac{1}{d m^{2}\left(d-1+m^{2}\right)}\left[(d-1)(1+u)^{2} G^{\prime \prime}+\left(d^{2}-1-d m^{2}\right)(1+u) G^{\prime}-m^{2}\left(d^{2}-1\right) G\right] .
$$

Повторное использование уравнения (20) приводит к выражению для $Y$ :

$$
\begin{aligned}
Y & =\frac{1}{d m^{2}\left(d-1+m^{2}\right)} \times \\
& \times\left[(d-1)(1+u)^{2} G^{\prime \prime \prime}+\left(2 d^{2}+d-3\right)(1+u) G^{\prime \prime}+\left(\left(d^{2}-1\right)(d+1)+m^{2}\right) G^{\prime}\right] .
\end{aligned}
$$

Дважды интегрируя (15) с учетом (20), получаем

$$
\begin{aligned}
(d-1) Z^{\prime}= & \frac{d-1}{m^{2}}(1+u)^{2} G^{\prime \prime}+\frac{d^{2}-1+2 m^{2}}{m^{2}}(1+u) G^{\prime}+ \\
& +2(d-1) G+(d-1)(1+u) X^{\prime}+2\left(d-1+m^{2}\right) X
\end{aligned}
$$

После подстановки найденного решения для $X$ и последующего интегрирования мы приходим к следующей формуле:

$$
\begin{aligned}
Z= & \frac{1}{d m^{2}\left(d-1+m^{2}\right)}\left[(d-1)(1+u)^{3} G^{\prime \prime}+2\left(d^{2}-1+m^{2}\right)(1+u)^{2} G^{\prime}+\right. \\
& \left.+\left((d-1)\left(d^{2}-1\right)+m^{2}(2 d-1)\right)\left((1+u) G-q^{\prime}\right)\right]-\frac{2}{d} q^{\prime}
\end{aligned}
$$

где

$$
q^{\prime \prime}(u)=G(u), \quad q(\infty)=q^{\prime}(\infty)=0 .
$$

Объединяя уравнения (15), (16) и (18) и принимая во внимание (19), находим

$$
\begin{aligned}
& (1+u)^{2} G^{\prime \prime}+(d+3)(1+u) G^{\prime}+2 G^{\prime \prime}+4(d-1) G+ \\
& +m^{2}(1+u) X^{\prime}+2 m^{2} X+(d-1) H^{\prime \prime}-m^{2} Z^{\prime}=0 .
\end{aligned}
$$


Разрешая уравнения (30) и (32) относительно $Z^{\prime}$, можно выразить $H^{\prime \prime}$ через $G$ и $X$ следуюшим образом:

$$
\begin{aligned}
H^{\prime \prime}=\frac{2 m^{4}}{(d-1)^{2}} & X-\frac{2}{(d-1)^{2}} \times \\
\times & {\left[(d-1) G^{\prime \prime}+\left(d-1-m^{2}\right)(1+u) G^{\prime}+(d-1)\left(2(d-1)-m^{2}\right) G\right] . }
\end{aligned}
$$

Наконец, подставляя выражение (28) в (33) и выполняя интегрирование, получаем

$$
\begin{aligned}
H= & \frac{2}{d(d-1)\left(d-1+m^{2}\right)}\left[m^{2}(1+u)^{2} G-d\left(d-1+m^{2}\right) G+\right. \\
& +\left((d-3) m^{2}-d(d-1)\right)(1+u) q^{\prime}+ \\
& \left.+\left(2 d(d-1)(2-d)-m^{2}\left(d+d^{2}+m^{2}-4\right)\right) q\right]
\end{aligned}
$$

Таким образом, полное решение для пропагатора массивного тензорного поля дается соотношением (13), где скалярные функции $G, X, Y, Z$ и $H$ определяются формулами $(23),(28),(29),(31)$ и (34), соответственно. Заметим, что в пределе $m^{2} \rightarrow 0$ функции $G$ и $H$ совпадают с калибровочно-инвариантной частью гравитонного пропагатора [12], в то время как функции $X, Y, Z$ сингулярны в этом пределе.

\section{3. СТРУКТУРА ПРОПАГАТОРА \\ НА ГРАНИЦЕ ПРОСТРАНСТВА AdS}

Полученное выражение для пропагатора массивного тензорного поля позволяет проанализировать его связь с функцией Грина данного поля, а также с двухточечной корреляционной функцией соответствуюшего оператора в граничной КТП. Напомним, что, как было замечено в работе [17], сушествует довольно простая связь между пропагатором скалярного поля $G\left(x_{1}, x_{2}\right)$ на пространстве $\mathrm{AdS}$ и функцией Грина $K\left(x_{1}, \overrightarrow{\mathbf{x}}_{2}\right)$ :

$$
K\left(x_{1}, \overrightarrow{\mathbf{x}}_{2}\right)=\lim _{x_{20} \rightarrow 0} C x_{20}^{-\Delta} G\left(x_{1}, x_{2}\right) .
$$

Здесь $C=2 \Delta-d$ и $\Delta=d / 2+\sqrt{(d / 2)^{2}+m^{2}}$ [18]. Таким образом, возникает возможность извлечения $n$-точечной корреляционной функции в граничной КТП исходя из соответствуюшего пропагатора, вычисленного на пространстве AdS. Для этого следует умножить пропагатор на подходящую степень $x_{i 0}$ и взять предел, при котором $x_{i 0}$ стремится к нулю:

$$
\left\langle\mathcal{O}\left(\overrightarrow{\mathbf{x}}_{1}\right) \ldots \mathcal{O}\left(\overrightarrow{\mathbf{x}}_{n}\right)\right\rangle=C_{1} \ldots C_{n} \lim _{x_{i 0} \rightarrow 0}\left(\prod_{i=1}^{n} x_{i 0}^{-\Delta_{i}}\right) G\left(x_{1}, \ldots, x_{n}\right) .
$$

Данное соотношение предполагает естественную идентификацию между квантовым полем $\hat{\phi}(x)$ на пространстве AdS и соответствуюшим ему оператором $\mathcal{O}(\overrightarrow{\mathbf{x}})$ в граничной КТП [19]. А именно, мы имеем

$$
\mathcal{O}(\overrightarrow{\mathbf{x}})=C \lim _{x_{0} \rightarrow 0} x_{0}^{-\Delta} \hat{\phi}(x) .
$$


Происхождение множителя $x_{0}^{-\Delta}$ в формуле (36) имеет следуюшее объяснение. Поле $\hat{\phi}(x)$ инвариантно относительно преобразований изометрий пространства AdS, в то время как оператор $\mathcal{O}(\overrightarrow{\mathbf{x}})$ преобразуется как примарное поле конформной размерности $\Delta$. Заметим, что преобразование $\mathcal{O}(\overrightarrow{\mathbf{x}})$ индуцируется изометриями пространства AdS в пределе $x_{0} \rightarrow 0$. Используя параметризацию $(1)$, отмеченные изометрии приводим к следуюшему виду:

$$
\begin{aligned}
\delta x^{i} & =-\xi^{i}-x_{0}^{2} \Lambda_{\mathrm{K}}^{i}, \\
\delta x_{0} & =x_{0}\left(2 x_{j} \Lambda_{\mathrm{K}}^{j}-\lambda_{\mathrm{D}}\right) \equiv x_{0} \Lambda_{\mathrm{D}},
\end{aligned}
$$

где

$$
\xi^{i}=a^{i}+\lambda_{\mathrm{M}}^{i j} x_{j}+\lambda_{\mathrm{D}} x^{i}+\left(\overrightarrow{\mathrm{x}}^{2} \Lambda_{\mathrm{K}}^{i}-2 x^{i} x_{j} \Lambda_{\mathrm{K}}^{j}\right) .
$$

Здесь параметры $a^{i}, \lambda_{\mathrm{M}}^{i j}, \lambda_{\mathrm{D}}$ и $\Lambda_{\mathrm{K}}^{i}$ соответствуют сдвигам, лоренцевым врашениям и бустам, дилатациям и специальным конформным преобразованиям, соответственно. Скалярное поле инвариантно относительно перечисленных преобразований, т.е. $\delta \hat{\phi}(x)=0$, в то время как поле $x_{0}^{-\Delta} \hat{\phi}(x)$ преобразуется по правилу

$$
\delta\left(x_{0}^{-\Delta} \hat{\phi}(x)\right)=\delta\left(x_{0}^{-\Delta}\right) \hat{\phi}(x)+x_{0}^{-\Delta} \delta \hat{\phi}(x)=-\Delta \Lambda_{\mathrm{D}} x_{0}^{-\Delta} \hat{\phi}(x) .
$$

Таким образом, мы установили, что $x_{0}^{-\Delta} \hat{\phi}(x)$ является примарным полем конформной размерности $\Delta$. В пределе $x_{0} \rightarrow 0$ преобразования (37) сводятся к обычным конформным отображениям плоского пространства и $x_{0}^{-\Delta} \hat{\phi}(x)$ становится оператором конформной размерности $\Delta$.

Идентификация (36) незначительно модифицируется в случае, когда рассматриваются поля $\phi_{s}$ с ненулевым спином $s$, и принимает форму

$$
\mathcal{O}_{s}(\overrightarrow{\mathbf{x}})=C \lim _{x_{0} \rightarrow 0} x_{0}^{-(\Delta-[s])} \hat{\phi}_{s}(x),
$$

где $[n]$ обозначает целую часть числа $n$. Данное обстоятельство подсказывает следующую модификацию соотношения (35):

$$
\left\langle\mathcal{O}_{s_{1}}\left(\overrightarrow{\mathbf{x}}_{1}\right) \ldots \mathcal{O}_{s_{n}}\left(\overrightarrow{\mathbf{x}}_{n}\right)\right\rangle=C_{1} \ldots C_{n} \lim _{x_{i 0} \rightarrow 0}\left(\prod_{i=1}^{n} x_{i 0}^{-\Delta_{i}+\left[s_{i}\right]}\right) G_{\left\{s_{i}\right\}}\left(x_{1}, \ldots, x_{n}\right) .
$$

Покажем теперь, как реализуется данная формула в случае массивного тензорного поля, рассмотренного ранее.

Из формулы (2) следует, что хордовое расстояние $u$ расходится в пределе $y_{0} \rightarrow 0$, так что для вычисления правой части соотношения $(40)$, где $G_{\left\{s_{i}\right\}}\left(x_{1}, x_{2}\right)$ было найдено в предыдушем разделе, необходимо знать поведение функции $G(u)$ в пределе, когда аргумент стремится к бесконечности. Принимая во внимание разложение Тейлора для гипергеометрической функции [20], находим

$$
G=\frac{1}{2 \pi^{d / 2}}\left(\frac{\Gamma(\Delta)}{\Gamma(\Delta-d / 2+1)}\left(\frac{2}{z}\right)^{\Delta}+\frac{\Gamma(\Delta+2)}{\Gamma(\Delta-d / 2+2)}\left(\frac{2}{z}\right)^{\Delta+2}\right)+O\left(\frac{1}{z}\right)^{\Delta+4} .
$$


Теперь воспользуемся формулами (14), а также зависимостью $X, Y, Z$ и $H$ от функции $G$ и разложим последнюю в ряд Тейлора, принимая во внимание (41). В результате получим

$$
\begin{aligned}
& A(u)=-\frac{(\Delta+1)}{2^{\Delta} \pi^{d / 2} d} \frac{\Gamma(\Delta-1)}{\Gamma(\Delta-d / 2+1)} z^{-\Delta}+O\left(z^{-\Delta-2}\right), \\
& B(u)=\frac{\Delta+1}{2^{\Delta} \pi^{d / 2}} \frac{\Gamma(\Delta-1)}{\Gamma(\Delta-d / 2+1)} z^{-\Delta-2}+O\left(z^{-\Delta-4}\right), \\
& C(u)=\frac{\Delta+1}{2^{\Delta+1} \pi^{d / 2}} \frac{\Gamma(\Delta-1)}{\Gamma(\Delta-d / 2+1)} z^{-\Delta}+O\left(z^{-\Delta-2}\right), \\
& D(u)=\frac{(\Delta+1)}{2^{\Delta} \pi^{d / 2} d} \frac{\Gamma(\Delta-1)}{\Gamma(\Delta-d / 2+1)} z^{-\Delta-2}+O\left(z^{-\Delta-4}\right), \\
& E(u)=-\frac{(\Delta+1)}{2^{\Delta+1} \pi^{d / 2}} \frac{\Gamma(\Delta-1)}{\Gamma(\Delta-d / 2+1)} z^{-\Delta-1}+O\left(z^{-\Delta-3}\right) .
\end{aligned}
$$

Напомним, что $z=1+u$. Подставляя полученные разложения в формулу (11) и используя явные выражения для битензоров (12), а также соотношения (3), находим

$$
\begin{aligned}
\lim _{y_{0} \rightarrow 0} y_{0}^{-(\Delta-2)} G_{\mu \nu ; i^{\prime} j^{\prime}}(x, y)=\frac{1}{\Delta-d / 2} \frac{\Delta+1}{\Delta-1} \frac{\Gamma(\Delta)}{\pi^{d / 2} \Gamma(\Delta-d / 2)} \times \\
\times \frac{x_{0}^{\Delta-2}}{\left(x_{0}^{2}+(\overrightarrow{\mathbf{x}}-\overrightarrow{\mathbf{y}})^{2}\right)^{\Delta}} J_{\mu k}\left(x_{0}, \overrightarrow{\mathbf{x}}-\overrightarrow{\mathbf{y}}\right) J_{\nu l}\left(x_{0}, \overrightarrow{\mathbf{x}}-\overrightarrow{\mathbf{y}}\right) P_{k l ; i^{\prime} j^{\prime}},
\end{aligned}
$$

где

$$
\begin{gathered}
J_{\mu \nu}(x)=\delta_{\mu \nu}-\frac{2 x_{\mu} x_{\nu}}{x^{2}}, \\
P_{k l ; i^{\prime} j^{\prime}}=\frac{1}{2} \delta_{k i^{\prime}} \delta_{l j^{\prime}}+\frac{1}{2} \delta_{k j^{\prime}} \delta_{l i^{\prime}}-\frac{1}{d} \delta_{k l} \delta_{i^{\prime} j^{\prime}}
\end{gathered}
$$

Сравнивая выражение (42) с соответствующей функцией Грина для массивного тензорного поля, полученной в импульсном представлении в работе [14], приходим к следующему соотношению:

$$
K_{\mu \nu ; i^{\prime} j^{\prime}}(x, \overrightarrow{\mathbf{y}})=\left(\Delta-\frac{d}{2}\right) \lim _{y_{0} \rightarrow 0} y_{0}^{-(\Delta-2)} G_{\mu \nu ; i^{\prime} j^{\prime}}(x, y) .
$$

Следует отметить, что функция Грина $K_{\mu \nu ; i^{\prime} j^{\prime}}(x, \overrightarrow{\mathbf{y}})$ может быть независимо получена путем решения уравнения (6) с внешним источником $T_{i j}(\overrightarrow{\mathbf{x}})$, зависяшим от координаты $\overrightarrow{\mathbf{x}}$ границы пространства AdS. Таким образом, соотношение (43) есть не что иное, как пропагатор поля спина $s=2$ с граничным условием Дирихле. В пределе $x_{0} \rightarrow 0$ тензор $G_{\mu \nu ; \mu^{\prime} 0}$ стремится к нулю быстрее, чем $G_{\mu \nu ; i^{\prime} j^{\prime}}$. Наконец, для того чтобы установить соответствие с формулой (40), рассмотрим предел при $x_{0} \rightarrow 0$ функции $K_{\mu \nu ; i^{\prime} j^{\prime}}$. Данный предел, определяющий двухточечную корреляционную функцию, равен

$$
\begin{aligned}
& \left(\Delta-\frac{d}{2}\right) \lim _{x_{0} \rightarrow 0} x_{0}^{-(\Delta-2)} K_{i j ; i^{\prime} j^{\prime}}(x, \overrightarrow{\mathbf{y}})= \\
& \quad=\frac{(\Delta-d / 2)(\Delta+1) \Gamma(\Delta)}{\pi^{d / 2}(\Delta-1) \Gamma(\Delta-d / 2)} \frac{1}{|\overrightarrow{\mathbf{x}}-\overrightarrow{\mathbf{y}}|^{2 \Delta}} J_{i k}(\overrightarrow{\mathbf{x}}-\overrightarrow{\mathbf{y}}) J_{j l}(\overrightarrow{\mathbf{x}}-\overrightarrow{\mathbf{y}}) P_{k l ; i^{\prime} j^{\prime}}
\end{aligned}
$$


Полученное выражение полностью воспроизводит результат работы [14]. Используя формулу (43), находим

$$
\left\langle\mathcal{O}_{i j}(\overrightarrow{\mathbf{x}}) \mathcal{O}_{i^{\prime} j^{\prime}}(\overrightarrow{\mathbf{y}})\right\rangle=\left(\Delta-\frac{d}{2}\right)^{2} \lim _{x_{0}, y_{0} \rightarrow 0} x_{0}^{-\Delta+2} y_{0}^{-\Delta+2} G_{i j ; i^{\prime} j^{\prime}}(x, y) .
$$

Таким образом, идентификация между массивным тензорным полем, распространяющимся на пространстве AdS, и примарным киральным оператором $\mathcal{O}_{i j}(\overrightarrow{\mathbf{x}})$ принимает следующий вид:

$$
\mathcal{O}_{i j}(\overrightarrow{\mathbf{x}})=\left(\Delta-\frac{d}{2}\right) \lim _{x_{0} \rightarrow 0} x_{0}^{-\Delta+2} \phi_{i j}(x)
$$

Наиболее изученным примером $\mathrm{AdS} / \mathrm{CFT-соответствия} \mathrm{является} \mathrm{случай,} \mathrm{когда} \mathrm{фоно-}$ вым пространством служит $A d S_{5} \times S^{5}$. Тогда $d=4$ и $m^{2}=k(k+4)$, а выражение (44) правильно воспроизводит двухточечный коррелятор примарного кирального поля спина $s=2$ и конформной размерности $k+4$ в $\mathcal{N}=4 S U(N)$-суперсимметричной теории Янга-Миллса.

\section{4. ЗАКЛЮЧЕНИЕ}

В настоящей работе мы построили пропагатор массивного симметрического тензорного поля ранга 2 на евклидовом продолжении пространства AdS. B вычислениях использовался тот факт, что пропагатор на пространстве $\mathrm{AdS}$ инвариантен относительно изометрий пространства и, следовательно, зависит только от одной скалярной переменной - хордового расстояния в пространстве вложения.

Также мы рассмотрели поведение пропагатора в пределе, когда тензорное поле принимает значение на границе пространства AdS. Мы показали, что полученные в этом пределе выражения совпадают с функцией Грина и двухточечной корреляционной функцией в граничной КТП. Данное обстоятельство предоставляет еще одно свидетельство в пользу того, чтобы рассматривать операторы граничной КТП как некоторые пределы квантовых полей, определенных на пространстве AdS.

Процедура нахождения двухточечной корреляционной функции в граничной КТП, использованная в настоящей работе, представляется более естественной, нежели стандартная процедура, требуюшая решения граничной задачи Дирихле с последуюшим вычислением гравитационного действия на массовой поверхности. Наш подход позволяет избежать как проблемы расходимостей, так и сложности нахождения граничных членов в некоторых случаях. Также нам не пришлось прибегать к так называемой $\epsilon$-регуляризации, которая явно нарушает конформную инвариантность и значительно усложняет вычисления. Полученный с помощью нашей процедуры коррелятор автоматически удовлетворяет конформным тождествам Уорда.

В заключение заметим, что полученный пропагатор может быть использован для исследований в области $\mathrm{AdS} / \mathrm{CFT-соответствия.} \mathrm{В} \mathrm{частности,} \mathrm{для} \mathrm{определения} n$-точечной корреляционной функции, построенной из операторов $\mathcal{O}_{i j}(\overrightarrow{\mathbf{x}})$ в граничной КТП, требуется использование пропагатора массивного симметрического тензорного поля. 
Благодарности. Автор благодарит Г.Э. Арутюнова и С.А. Фролова за помощь при постановке задачи, а также О.А. Рычкова за ценные обсуждения во время работы над статьей. Эта работа была частично поддержана грантами РФФИ № 99-01-00193 и 01-01-06093.

\section{Список литературы}

[1] O. Aharony, S. S. Gubser, J. Maldacena, H. Ooguri, Y. Oz. Phys. Rep. 2000. V. 323. P. 183; hep-th/9905111.

[2] C. Fronsdal. Phys. Rev. D. 1974. V. 10. P. 589

[3] C. P. Burgess, C. A. Lutken. Phys. Lett. B. 1985. V. 153. P. 137.

[4] C. Dullemond, E. van Beveren. J. Math. Phys. 1985. V. 26. P. 2050

[5] C. P. Burges, D.Z. Freedman, S. Davis, G. W. Gibbons. Ann. Phys. 1986. V. 167. P. 285.

[6] B. Allen, T. Jacobson. Commun. Math. Phys. 1986. V. 103. P. 669.

[7] B. Allen, M. Turyn. Nucl. Phys. B. 1987. V. 292. P. 813.

[8] M. Turyn. J. Math. Phys. 1990. V. 31. P. 669.

[9] I. Antoniadis, E. Mottola. J. Math. Phys. 1991. V. 32. P. 1037.

[10] A. A. Tseytlin, Hong Liu. Phys. Rev. D. 1999. V. 59. P. 086002; hep-th/9807097.

[11] E. D'Hoker, D. Z. Freedman. Gauge boson exchange in $A d S_{d+1}$. hep-th/9809179.

[12] E. D'Hoker, D.Z. Freedman, S. D. Mathur, A. Matusis, L. Rastelli. Nucl. Phys. B. 1999. V. 544. P. 612; hep-th/9902042.

[13] H. J. Kim, L. J. Romans, P. van Nieuwenhuizen. Phys. Rev. D. 1985. V. 32. P. 389.

[14] A. Polishchuk. JHEP. 1999. V. 9907. P. 007; hep-th/9905048.

[15] W. Mück, K. S. Viswanathan. Phys. Rev. D. 1998. V. 58. P. 106006; hep-th/9805145.

[16] I. L. Buchbinder, V. A. Krykhtin, V.D. Pershin. Phys. Lett. B. 1999. V. 466. P. 216; hep-th/9908028.

[17] T. Banks, M. Douglas, G. Horowitz, E. Martinec. AdS dynamics from conformal field theory. hep-th/9808016; U. Chicago preprint EFI-98-30. Chicago: Chicago Univ., 1998.

[18] I. R. Klebanov, E. Witten. Nucl. Phys. B. 1999. V. 556. P. 89; hep-th/9905104.

[19] J. Polchinski. S-matrices from AdS spacetime. hep-th/9901076.

[20] Г. Бейтмен, А. Эрдейи. Высшие трансцендентные функции. Т. 1, 2. М.: Наука, 1973, 1974. 